\title{
Clinical and Refractive Outcomes of Toric Phakic Implantable Collamer lens Implantat for correction of myopic astigmatism
}

\author{
Suzan Amana *
}

\begin{abstract}
Background: Currently there are four general approaches to correct refractive errors: refractive corneal surgery, crystalline lens surgery and implantation of an intraocular lens in anterior or posterior chamber.

Objective: To evaluate the predictability, safety and stability of toric phakic implantable collamer lens implantation to correct moderate to high myopic astigmatism. in Eye Specialty Private Hospital, Baghdad, Iraq.

Type of the Study: a prospective non randomize intervention study.

Methods: 60 eyes of 40 patients underwent implantation of a toric implantable collamer lens (V4c design).Mean spherical refraction was -11.32 diopter (D) \pm 3.17 (SD) with range from -6.00 to $-18.00 \mathrm{D}$ and the mean cylinder was $-2.61(D) \pm 1.16$ with range from -1.00 to $-5.50 \mathrm{D}$. The outcome measures that evaluated during a 12 months follow-up period include UDVA, refractive outcomes, CDVA, vault and adverse events.

Results: At 12 months postoperatively the mean Snellen decimal UDVA was $0.77 \pm 0.23$ and mean CDVA was $0.80 \pm 0.21$, with an efficacy index 1.16 . Twenty nine eyes $(48.33 \%)$ showed gain in CDVA with safety index
\end{abstract}

1.21.The treatment was highly predictable for spherical equivalent and astigmatic component.The mean SE dropped from $(-12.63 \mathrm{D} \pm 3.11)$ to $(-0.11 \mathrm{D} \pm 0.20)$ with 58 eyes within $\pm 0.50 \mathrm{D}$ and 60 eyes with $\pm 1.00 \mathrm{D}$ of target correction. For achieved cylinder 60 eyes (100\%) had $\leq 0.50 \mathrm{D}$ and 51 eyes (85\%) had $\leq 0.25 \mathrm{D}$ with strong positive linear correlation between achieved and expected cylinder $(r=0.94)$

Conclusions: The results of the present study support safety, efficacy, predictability of toric implantable collamer lens implantation to treat moderate to high myopic astigmatism.

Key words: PIOL: phakic intraocular lens, ICL: Implantable Collamer Lens.

Abbreviation: UDVA: uncorrected distance visual acuity, CDVA: corrected distance visual acuity, SE: spherical equivalent and $A C D$ : anterior chamber depth.

\section{Al-Kindy College Medical Journal 2017: Vol.13 No.1 Page: $70-75$}

${ }^{*} \mathrm{MBChB}$, FICMS (Oph.)- Al-Kindy Medical CollegeBaghdad University

Received $15^{\text {th }}$ Feb 2017, accepted in final $30^{\text {th }}$ March 2017

Corresponding to Assistant Prof. Dr. Suzan Amana
L aser corneal refractive surgery has been used to correct wide range of refractive errors and proved to be effective and safe in most cases. Despite the use of highly optimized and customized laser treatment such as wave front -guided, aspheric and topoguided ablation physical limitations of corneal thickness, curvature and tissue remodeling limit the indication for a safe corneal refractive procedure.Moreover, the optical quality of outcome may not be as good as desired especially when treat high refractive errors. When kerato refractive procedure is not appropriate approach, either phakic intraocular lens implantation or refractive lens exchange (RLE) with IOL implantation should considered.One of the main concerns about RLE in high myopic eye is increased risk of retinal detachment especially in younger patient and in eye with long axial length $>26 \mathrm{~mm}^{1 / 2}$. RLE leads to loss of accommodation and should be avoided when natural lens is still functioning .In the absence of contraindication PIOL implantation is the best approach in young patient with moderate to high refractive errors and in those who have contraindication to keratorefractive procedure.
Advantages of PIOL implantation include: it maintains accommodation and is reversible. The Visian Implantable Collamer Lens is a PIOL designed to be placed in the posterior chamber, just behind the iris with the haptic zone resting in the ciliary sulcus ${ }^{3}$.Previous studies have reported that implantation of this PIOL is a safe and effective treatment option in the refractive correction of myopia ${ }^{4^{\prime} 5}$,hyperopia ${ }^{6 / 7}$, astigmatism ${ }^{8 / 9}$ and in patient who may not be suitable candidate for corneal reshaping procedures ${ }^{10^{\prime} 11}$. The reported complications of previous model of this ICL include: anterior sub capsular cataract ${ }^{12}{ }^{-}{ }^{15}$, increase intraocular pressure $(\mathrm{IOP})^{16}$, endothelial cell loss ${ }^{17}$,pigment dispersion ${ }^{18}$ and pupillary block ${ }^{19^{\prime 20}}$. Currently, the model V4c Implantable Collamer Lens PIOL was designed with central hole of $0.36 \mathrm{~mm}$ to overcome the above mentioned disadvantages. The purpose of the present study was to assess the clinical and refractive outcomes in patients who had toric phakic ICL implantation of the V4c model to correct moderate to high myopic astigmatism over a 12 months follow -up period. 


\section{Methods:}

Enrollment and Baseline : This prospective study comprised 60 eyes of 40 patients with age range from $20 y$-38ys, twenty two were women , and 18 were men with refractive sphere range from -6.00 to $-18.00 \mathrm{D}$ and refractive cylinder from -1.00 to $-5.50 \mathrm{D}$. Table 1 shows the preoperative patients demographic data and the size of implanted lenses.

Inclusion criteria were best corrected distance visual acuity (CDVA) of 20/40 or better, stable refraction and clear central cornea.Exclusion criteria include age $<20 y s$, previous corneal refractive surgery ,anterior chamber depth of less than $2.8 \mathrm{~mm}$,corneal endothelial cell density less than $2000 \mathrm{cell} / \mathrm{mm}^{2}$, mesopic pupil larger than $7.0 \mathrm{~mm}$,cataract, history of glaucoma ,retinal detachment, macular degeneration or retinopathy ,neuro-ophthalmic disease and a history of ocular inflammation.

All patients provided written informed consent after the nature and possible consequences of the study were explained fully.

Preoperative evaluation: Before surgery, patients had a full ophthalmologic examination including : UDVA, CDVA, manifest and cycloplegic refractions,slit lamp evaluation ,Goldman applanation tonometry , gonioscopy, ultrasonic corneal pachymetry (Tomey SP100 ), and corneal topography(Sirius),central endothelial cell count (ECC), binocular indirect ophthalmoscopy through dilated pupil and white to white distance measured by caliper. The targeted refraction in all patients was emmetropia.

Intraocularlens:The V4c Implantable Collamer Lens posterior chamber PIOL is made from Collamer, a biocompatible hydrophilic copolymer of collagen and hydroxylethyl methacrylate with an ultraviolet light filtering chromophore. This model is designed to correct myopia with or without astigmatism .It has plate- haptic design with central convex- concave optical zone and incorporates forward vault to minimize contact of the PIOL with the central anterior capsule of crystalline lens .A central hole of $0.36 \mathrm{~mm}$, the KS- Aqua port, defines the new design of $\mathrm{V} 4 \mathrm{C}$ PIOL. The hole allows more neutral flow of the aqueous humor (central flow technology) eliminating the need to perform preoperative or intra operative Nd-YAG peripheral iridectomies. The PIOL is available in 4 overall lengths $(12.1 \mathrm{~mm}, 12.6 \mathrm{~mm}$, $13.2 \mathrm{~mm}$ and $13.7 \mathrm{~mm}$ ) and a myopic power range -0.50 to $-18.00 \mathrm{D}$ and cylinder range -0.50 to $-6.00 \mathrm{D}$. The power of the PIOL was calculated using the PIOL power table software provided by the manufacturer; the table used a modified vertex formula. The targeted refraction was emmetropia in all cases. The size of the PIOL was also chosen by the manufacturer based on the horizontal white-white distance measured by caliper and ACD that measured with thecorneal topography.
Surgical technique:All surgeries were performed by the same surgeon at Eye Specialty Private Hospital, Baghdad, Iraq. Before the surgery a dotes marked at the slit lamp as reference for $0^{\circ}-180^{\circ}$ axis of the eye then serial tropicamide and phenylephrine eye drops instillation for pupil dilatation. Five minutes before surgery ,povidine -iodine $5 \%$ was applied . The surgical procedure was performed through a singleplane 3.0 to $3.2 \mathrm{~mm}$ corneal incision centered temporally under topical anesthesia .The anterior chamber filled with sodium hyaluronate $1 \%$, which was completely removed at the end of surgery. The PIOL was inserted with the use of an injector cartilage (staar surgical Co.).No preoperative or intra operative peripheral iridectomies were performed in any case. Toric ICL is manufactured to minimize rotation and required the surgeon to rotate the toric ICL no more than $22.5^{\circ}$ from the horizontal meridian. Each toric ICL comes with guide demonstrating the amount and direction of rotation from the horizontal axis required of the toric ICL to exactly align the toric ICL cylinder axis to the patients' required cylinder correction.

Postoperative topical therapy included tobramycin and dexamethasone $0.1 \%$ (tobradex) eye drop 4 times for 7 days .In case of bilateral implantation, the second eye was operated on within 1 week after the $1^{\text {st }}$ surgery.

Post operative assessment: Postoperative follow-up visits were scheduled at 3 ,and 24 hours(early postoperative) ; 1 week ; and 1,3,6 and 12months .The examinations included UDVA,CDVA, refractive status , slit lamp examination ,tonometry , gonioscopy, subjective (slit lamp)and objective ( topography Scheimpflug image) vault assessment and fundoscopy. The vault between the crystalline lens and the PIOL was measured perpendicular to the lens apex or at the narrowest space between both.

Outcomes:Assessment of outcomes was based on preoperative versus postoperative UDVA and CDVA values (efficacy and safety respectively).The expected versus the achieved refractive out comes postoperatively (predictability) and adverse complications .The efficacy index (ratio of postoperative UDVA to preoperative CDVA) and the safety index (ratio of postoperative to preoperative CDVA) were also calculated.

Statistical analysis: Data analysis was performed using Minitab statistical software (version 13). Normality of data was checked by the Kolmogorov -Smirnov test. Statistical differences between preoperative and postoperative refractive and visual outcomes were analyzed with Two-sample T-Test.

All recorded visual acuity data were converted to Log MAR values. Differences with a $p$ value less than 0.05 were considered statistically significant. 
Results: This study evaluated 60 eyes of 40 patients .The mean age at the time of surgery was 26.07ys \pm 5 .32. At 12 months post surgery, the mean spherical error was $-0.08 \mathrm{D} \pm 0.17$ and the mean cylinder value was $-0.11 \mathrm{D} \pm 0.18$.

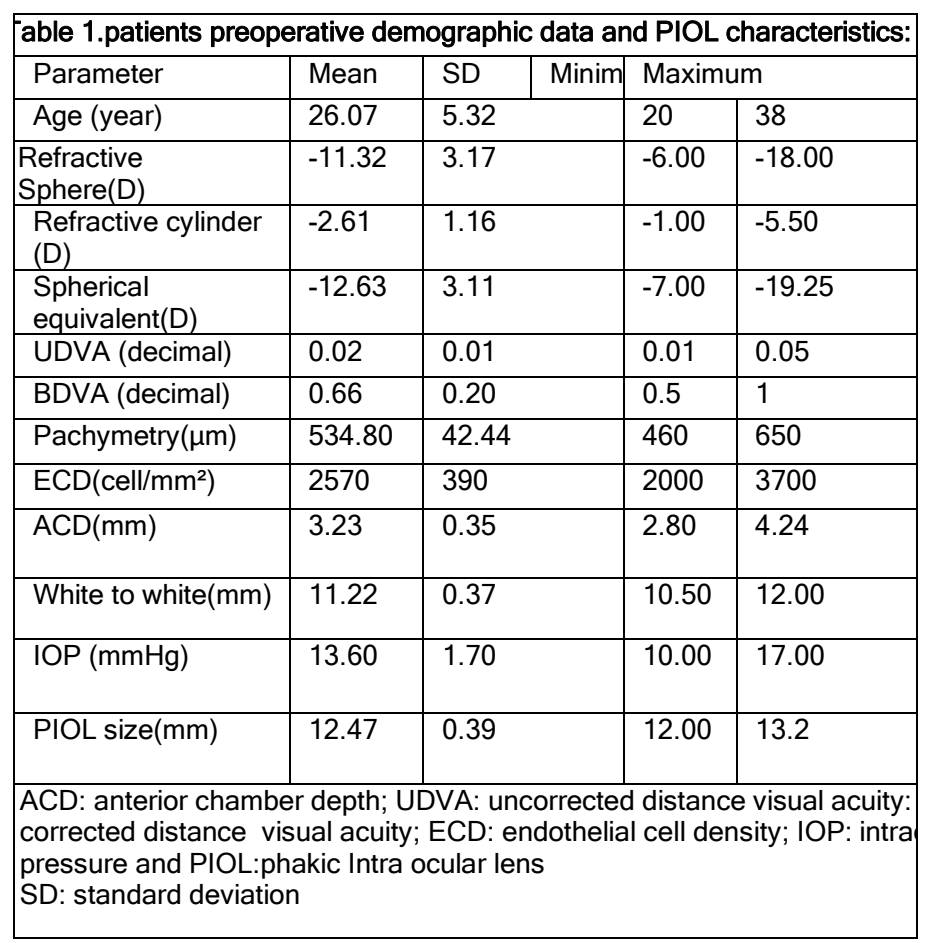

Efficacy: The mean postoperative LogMAR UDVA was $(0.15 \pm 0.09,0.14 \pm 0.04,0.13 \pm 0.14) 1$ month, 6 months and 12 months respectively. There was a statistically significant difference between the preoperative CDVA and 12 months postoperative LogMAR UDVA ( $p$ 0.007) .The UDVA was 0.3 ( 20/40 )or better in all eyes at all times after surgery .It was $0.00(20 / 20)$ in29 eyes $(48.33 \%)$ at 12 months post operatively . The efficacy index at 12 months was 1.16 (table2).

\begin{tabular}{|r|c|c|c|c|}
\hline \multicolumn{5}{|c|}{ Table 2: pre op CDVA versus postop UDVA } \\
\hline Parameter & Mean & SD & P value & $\begin{array}{l}\text { Efficacy } \\
\text { index }\end{array}$ \\
\hline Pre op. CDVA(decimal) & 0.66 & 0.20 & & \\
\hline Post op. UDVA(decimal) & 0.77 & 0.23 & 0.007 & 1.16 \\
& & & & \\
\hline
\end{tabular}

Predictability: Figure 1 shows the deviation of the achieved from the expected spherical equivalent SE. 12 months postoperatively, the achieved SE was close to emmetropia (mean $-0.11 \mathrm{D} \pm 0.20$ ) with 58 eyes $(96.66 \%$ ) within $\pm 0.50 \mathrm{D}$ and 60 eyes (100\%) within $\pm 1.00 \mathrm{D}$ of target correction. High correlation between attempted and achieved SE (with Pearson correlation between achieved and expected $S E=0.94, R^{2}=0.89$ and $p$ value 0.000).
No eye $(0.0 \%)$ preoperatively had refractive cylinder of $0.50 \mathrm{D}$, whereas 12 months post operatively 60 eyes $(100 \%$ )had $\leq 0.50 \mathrm{D}$ and 51 eyes $(85 \%) \leq 0.25 \mathrm{D}$ with strong linear correlation between achieved and expected cylinder (with Pearson correlation $=0.94$ and $p$ value 0.000 )

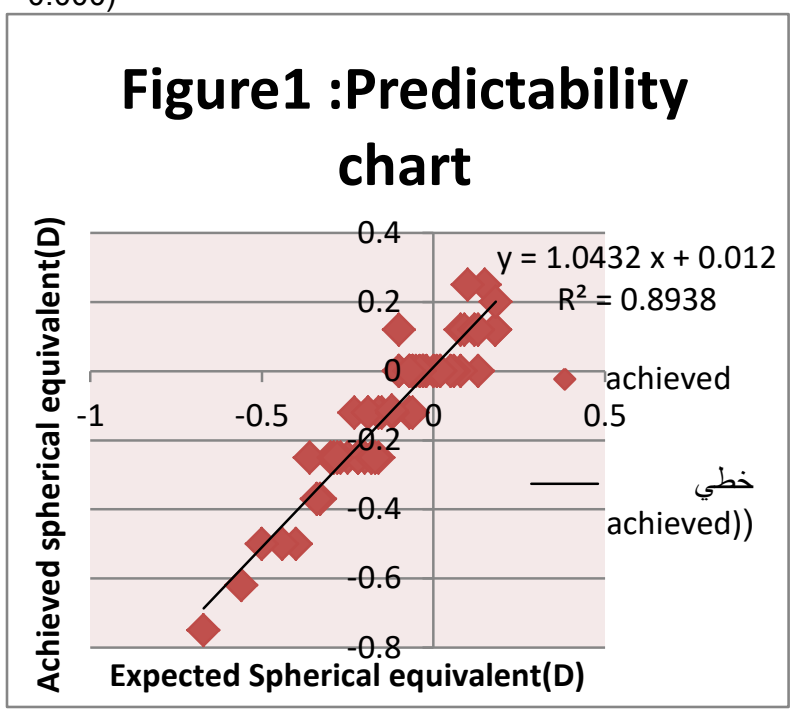

Safety: The mean postoperative LogMAR CDVA was ( $0.14 \pm 0.09,0.13 \pm 0.15, \quad 0.11 \pm 0.12)$ at 1 month,6months, and 12 months respectively .All eyes achieved CDVA of $0.3(20 / 40)$ ) or better after PIOL implantation, with 30 eyes(50\%) having a CDVA of 0.0 $(20 / 20)$ or better at 12 months of follow- up. There was significant improvement in LogMAR CDVA after surgery (p 0.000 ), no eye loss 1 or more lines, thirty one eyes $(51.67 \%)$ did not change from preoperatively ,five eyes( $8.33 \%$ ) gained 1 line , seventeen eyes $(28.33 \%)$ gained 2 lines and 7 eyes (11.67\%) gain 3 lines (figure 2). The safety index was 1.21 (table 3 ) at 12 months follow up

\begin{tabular}{|c|r|r|r|r|}
\hline Table3:preop versus postop CDVA \\
\hline Parameter & Mean & SD & P value & Safety index \\
\hline Preop. CDVA (decimal) & 0.66 & 0.20 & & \multirow{2}{*}{0.000} \\
\cline { 1 - 3 } Postop CDVA(decimal) & 0.80 & 0.21 & & 1.21 \\
\hline
\end{tabular}




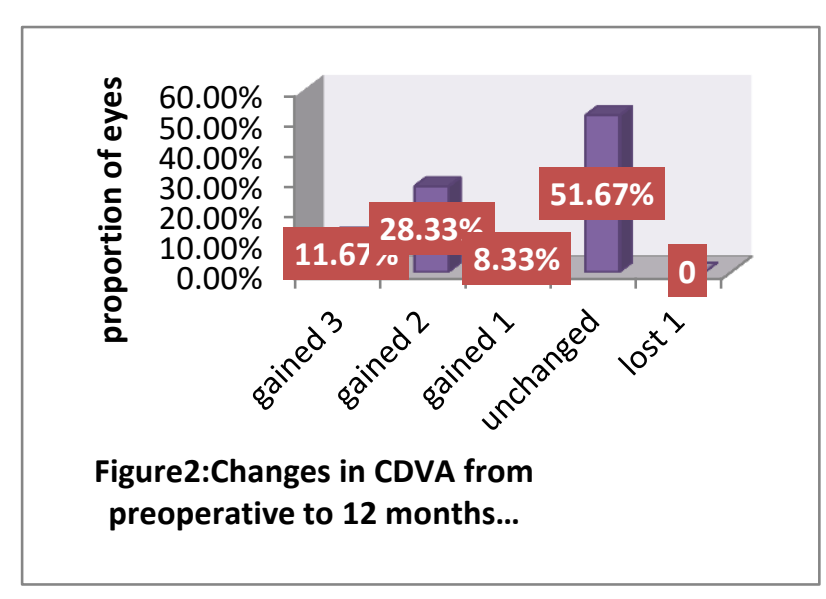

Stability: The mean preoperative SE was $-12.63 \mathrm{D} \pm$ 3.11. The mean postoperative SE was $-0.13 \mathrm{D} \pm 0.20$ at 1 month , $-0.12 \mathrm{D} \pm 0.18$ at 6 months and $-0.11 \mathrm{D} \pm 0.20$ at 12 months. The decrease in mean SE from preoperatively to 12 months postoperatively was statistically significant (P 0.000).

Intraocular pressure and vault: The mean IOP was 13.6 $\pm 1.7 \mathrm{mmHg}$ preoperatively. Postoperatively ,the mean IOP was $11.6 \pm 1.8 \mathrm{mmHg}$ in early postoperative period , $12.3 \pm 1.3 \mathrm{mmHg}$ at 1 month, $12.5 \pm 1.3 \mathrm{mmHg}$ at 6 months, and $12.4 \pm 1.5 \mathrm{mmHg}$ at 12 months .No significant rise in IOP $(>20 \mathrm{mmHg})$ occurred in any case during the 12 months follow up.

The mean postoperative vault was $575.8 \mu \mathrm{m} \pm 180.8$ at 1 week, $565.5 \pm 188.6 \mu \mathrm{m}$ at 1 month, $535.9 \mu \mathrm{m} \pm 202.9$ at 6 months, $485.7 \mu \mathrm{m} \pm 240.5$ at 12 months follow up. There was trend toward decrease in the mean objective vault over time. Adverse events and secondary surgeries: There were no complications during the surgical procedure. Postoperatively, no eye required PIOL explantation or repositioning, and there were no cases of decentration of the PIOL optic. No cataract formation, pigmentary glaucoma, pupillary block, or other visionthreatening complications occurred at any time during the 12 months follow up.

Discussion: The aim of the current study was to determine whether implantation of the toric Implantable Collamer Lens PIOL is safe, predictable, and effective for moderate to high myopic astigmatism correction and to analyze the benefits or disadvantage related to the new design.

In order to compare with previous studies that reporting data of the toric ICL, a 1year follow- up study showed that toric ICL (V4design) implantation is highly predictable, stable and safe up to 12 months post surgery. The majority of eyes maintained or improved their CDVA, which resulted in high safety index (1.14), more than $60 \%$ of eyes showed a gain in CDVA, only 1 eye lost 2 lines of CDVA at 12 months. In addition over $80 \%$ of eyes had CDVA of $20 / 25$ or better at 12 months and the percent of eyes with $20 / 20$ or better increased from $30 \%$ preoperatively to $70 \%$ after toric ICL implantation ${ }^{21}$. A 3ys follow-up study of V4 toric ICL implantation showed that the lens was good in all measures of safety, efficacy, predictability, and stability for correction of moderate to high myopic astigmatism throughout 3 years observation period ${ }^{22}$. Regard the new iridotomy free design V4c toric ICL a study showed good outcomes over 6 months follow- up period, it was limited to eyes with cylinder range ( 1 to $4.5 \mathrm{D}$ ) and myopia (spherical equivalent)between 6 to 18.25D. The mean refractive cylinder changed from 2.64 D preoperatively to $0.59 \mathrm{D}$ postoperatively .Baseline manifest refraction spherical equivalent changed from 13.18 to $0.00 \mathrm{D}$.Safety concern not identified ${ }^{23}$. The current study followed similar methodology of the previous study but in larger sample of patients who had

V4c PIOL implantation over longer period of follow up and for correction of higher astigmatic range from (-1.00 to- $5.50 \mathrm{D}$ ). It shows a statistically significant reduction in the mean refractive cylinder $(p 0.000)$ from $-2.61 \mathrm{D} \pm 1.16$ to $-0.11 \mathrm{D} \pm 0.18$. We obtained stable results and near emmetropia (mean SE -0.11D \pm 0.20 ). Fifty eight eyes $(96.66 \%)$ were within $\pm 0.50 \mathrm{D}$ and all eyes $100 \%$ within $\pm 1.00 \mathrm{D}$ of targeted correction , twenty nine ( $48.33 \%$ ) eyes showed a gain in CDVA with a high safety index (1.21) , twenty eyes (33.33\%) had postoperative UDVA better than the preoperative CDVA , thirty three eyes (55\%) had CDVA $20 / 25$ or better and the percent of eyes that had the CDVA 20/20 increased from $23.33 \%$ (14) eyes preoperatively to $50 \%$ (30)eyes at 12 months after toric ICL implantation. It is reasonable to believe that a central artificial hole in optic of the V4c Implantable Collamer Lens PIOL may deteriorate the optical quality of $\mathrm{PIOL}$ by ,for example , introducing halos or glare and consequently decreasing the visual quality of the patients. However ,a previous study by Shiratani et al ${ }^{24}$.that evaluated the modulation transfer function (MTF) of an Implantable Collamer Lens PIOL with and without a central hole reported similar MTF for both PIOL designs .Their results agreed with those reported in the vitro study of Uozato et al. ${ }^{25}$ In which small differences in the optical performance with negligible clinical effect were found with a PIOL with $0.36 \mathrm{~mm}$ central hole and a conventional PIOL .These outcomes suggest that the central hole in V4c Implantable Collamer Lens does not affect the optical quality and therefore the patient's visual quality .Our results agree with this considering our good visual acuity outcomes, which are similar to those reported in previous studies that evaluated the earlier version of this $\mathrm{PIOL}^{4^{\prime} 13^{\prime} 26}$. The central hole in the optic of $\mathrm{V} 4 \mathrm{C}$ Implantable Collamer Lens allows more natural aqueous humor circulation and Nd-YAG iridectomies are not necessary, eliminating the risks and complications 
of this procedure.In the present study no significant rise in IOP ( $>20 \mathrm{mmHg}$ ) was found in any case after the 12months follow -up even without preoperative or intra operative peripheral iridectomies However when we evaluate change in IOP over time and calculate variations between preoperative and postoperative values, we found that in most eyes, the IOP remained unchanged or showed a reduction of 1 to $2 \mathrm{mmHg}$ from preoperatively at each follow -up.

Several authors describe other concerns associated with PIOL implantation, such as anterior sub capsular opacities $^{12^{\prime 15^{\prime}} 27}$ and endothelial cell loss ${ }^{17}$. Cataract formation has been related to surgical trauma (early cataract) or to constant or intermittent PIOL -crystalline lens contact because of insufficient vaulting (late cataract formation) ${ }^{15^{\prime 2}}$. Because the central hole in the PIOL implanted in the current study improves the circulation of aqueous humor to the anterior crystalline lens surface we found no anterior sub capsular opacities over the follow up period. However it is important to control the amount of central vault after PIOL implantation .In the current study we found a trend toward a decrease in the mean vault .In most eyes, the vault was between $400-600 \mu \mathrm{m}$.

Conclusion: Based on the predictability, efficacy, and safety outcomes in our study, implantation of the toric V4c Implantable Collamer Lens PIOL is effective, predictable, safe and stable for the correction of moderate to high myopic astigmatism during the 12 months follow- up.

Recommendation: Our results agree with those reported with this lens previously, however, larger sample of patients and for longer duration of follow -up may be required to assess the safety and stability of this surgical procedure, particularly in terms of increased IOP.

\section{References:}

1.Gu"ell,Rodriguesz-Arenas AF,GrisO,MalecazeF,et al . Phacoemulsification of crystalline lens and implantation of intraocular lens for the correction of moderate and high myopia:4 years follow up .J Cataract Refract Surg 2003:29:34-8.

2. Fernandez-Vega L, Alfenso JF, VillacampT,et al .Clear lens extraction for the correction of high myopia . Ophthalmology 2003;110:2349-54.

3. Lovisolo CF, RenisteinDZ,et al. Phakic intraocular lenses .Surv Ophthalmol $2005 ; 50: 549-587$.

4. ICl in treatment of myopia (ITM) study group. United states Food and Drug Administration clinical trial of the implantable Collamer Lens (ICL) for moderate to high myopia; 3 years follow up .Ophthalmology 2004;111:1692.

5. Arne JL, Lesueur LC,et al .Phakic posterior chamber lenses for high myopia: functional and anatomical outcomes ,J Cataract Refract Surg 2000;26:369-374

6. Peasando PM, Ghringhello MP, Di Meglio G, et al. Posterior chamber phakic intraocular lens (ICL)for hyperopia :ten-year follow-up .J Cataract Refract Surg2007;33(9):1579-1584
7. Davidorft JM, Zaldivar R,Oscherow S, et al. Posterior chamber phakic intraocular lens for hyperopia of +4 to+11 diopters. J Refract Surg 1998;14:306-311

8. Alfonso JF ,Fernandez-Vega L,Fernandez p ,Gonzalez Meijome JM,et al. Collagen copolymer toric posterior chamber phakic intraocular lenses for myopic astigmatism ,one-year follow up .J Cataract Refract Surg 2010;36:568-576.

9.Sanders DR ,Schneider D ,Martin R ,Brown D,Dulaney $D$, VukichJ ,et al. Toric implantable collamer lens for moderate to high myopic astigmatism .Ophthalmology $2007 ; 114: 54-61$.

10.Alfenso JF, palaciosA,Montes -Mico R ,et al .Myopic phakic STAAR collamer posterior chamber intraocular lenses for keratoconus .J Refract Surg 2008;24 :867874.

11. Alfenso JF ,Lisa C, Abdel Hamid A, Montes -Mico R ,et al. Posterior chamber phakic intraocular lenses after penetrating keratoplasty .J Cataract Refract Surg 2009 ;35:1166-1173.

12. Sanchez -Galeana CA,Smith RJ,Sanders DR,Rodreguesz FX, et al. Lens opacities after posterior chamber phakic intraocular lens implantation .Ophthalmology $2003 ; 110: 781-785$.

13. Lackner B,Pieh $S$,Schmindinger $G$,Simader C ,Franz C,et al. Long term result of implantation of phakic posterior chamber intraocular lenses .J Cataract Refract Surg 2004;30:2296-2276.

14. Sander AD,et al. Anterior sub capsular opacities and cataracts 5 years after surgery in Visian Implantable collamer lens FDA trial .J Cataract refract Surg2008;24:566-570.

15 .Alfenso JF ,Lisa C,AbdelhamidA,FernandesP,Jorg J,et al. Three-year follow up of subjective vault following myopic implantable collamer lens implantation .Graefes Arch ClincExp Ophthalmol $2010 ; 248: 1827-1835$.

16. Sanchez -Galeana CA ,ZadokD,MontesM,Cortes $M A$, et al. Refractory intraocular pressure increase after phakic posterior chamber intraocular lens implantation .Am J Ophthalmol 2002;134:121-123.

17. Dejaco- Ruhswurm I,Scholz U, Pieh S, Hanselmayer $\mathrm{G}$,Lackner B,et al. Long -term endothelial changes in phakic eyes with posterior chamber intraocular lenses. J Cataract Refract Surg $2002 ; 28: 1589-1593$.

18.Brandt JD,Mockovak ME,Chayet A,et al. Pigmentary dispersion syndrome induced by a posterior chamber phakic refractive lens .Am J Ophthalmol 2001 ;131:260-163.

19. BylsmaSS,ZaltaAH,Foley E, Osher RH,et al .Phakic posterior chamber intraocular lens pupillary block .J Cataract Refract Surg 2002;28: 2222-2228.

20.Smallman DS,ProbstL, Rafuse PE .Pupillary block glaucoma secondary to posterior chamber Phakic intraocular lens implantation for high myopia .J Cataract Refract Surg 2004;30:905-907.

21.Erik L Mertens. Toric phakic implantable collamer lens for correction of astigmatism: 1 year outcomes. Clinical ophthalmology 2011; 5:369-375. 
22. Kamiya K,Shimiizu K,Kobashi $H$,Igarashi A,KomatsuM ,et al .Three- years follow up of posterior chamber Toric Phakic Intraocular lens Implantation for Moderate to High Myopic Astigmatism.PLos ONE 2013; 8(2):e56453.

23. Chang $\mathrm{J}$ et al. Toric implantable collamer lens for high myopic astigmatism Asian eyes .Ophthalmology 2009;116(12):2340-7

24. Shiratani T ,Shimzu K,Fujisawa K,UgaS,Nagano $\mathrm{K}$,et al.Crystalline lens changes in porcine eyes with implanted phakic IOL(ICL) with central hole .Graefes Arch ClinExp Ophthalmol 2008;246:719-728.

25. Uozato $H$,Shimzu K KawamoritaT,Ohmoto F,et al.Modulation transfer function of intraocular collamer lens with central hole .Graefes Arch ClinExp Ophthalmol 2011;249:1081-1085.
26. Alfenso JF. Baamonde B, Fernandez -Vega L, Fernandes $P$, et al. Posterior chamber collagen copolymer phakic intraocular lens to correct myopia: five -year follow up. J Cataract Refract Surg 2011; 37:873880

27.Gonvers $\mathrm{M}$, Bornet $\mathrm{C}$,Othenim -Girard $\mathrm{P}$,et al. Implantable contact for moderate to high myopia ;relationship of vaulting to cataract formation .J Cataract Refract Surg 2003 ;29:918-924.

28.Pineda -Fernandez A .Jaramillo $\mathrm{J}$.Vargas $\mathrm{J}$ ,Jaramillo $M$,et al .Phakic posterior chamber intraocular lens for high myopia .J Cataract Refract Surg $2004 ; 30$ :2277-2283. 